\title{
A Smart Watch for Women Security
}

\author{
Pheba Cherian, A. Deepak
}

\begin{abstract}
The paper describes a prototype of wearable a device enhanced with GSM technology for protecting women during physical harassment or robbery. This device is having the sensing unit, in the form of a watch and is responsible for monitoring the heartbeat of the individual. It is equipped with a finger print scanner, to wear and remove the device. It also consists of RTC (Real Time Clock) which will display the clock in the system. Heartbeat sensor is used to monitor the heartbeat of the women. If any harassment takes place to women, naturally heartbeat will increase then the wearable device sends the SMS to respective people or police whose numbers are fed in the system. It will send the messages only after a preset delay, so that error can be avoided, during the course of exercise or walking fast.
\end{abstract}

\section{INTRODUCTION}

Most of the women all over the world undergo much unethical physical harassment. This acquires a fast pace due to deficiency of a suitable surveillance system. The only notion lingering in every girl's mind is about safe travelling without restraints on the streets even during nights fearlessly.

In this device, we are using wearable device enhanced with GSM technology. The Wearable Device is having the sensing unit, in the form of a watch and is responsible for monitoring the heartbeat of the person. Once we place the finger in the finger print scanner, it will open the system. The system consists of RTC (Real Time Clock) which will display the clock in the system. Here we are using heartbeat sensor to measure the heartbeat of the patient. If any harassment takes place to women, naturally heartbeat will increase then the wearable device sends the SMS to respective people or police through GSM technology. The system will take 10 seconds delay to send the SMS to respective people. Once the user place the finger in the finger print scanner within 10 seconds, the system understands that the person is normal and stop the SMS alert to respective people.

Anand Jatti, Et.al[1], analyze, electrodermal activity and temperature of the body and also body position using an accelerometer, it is monitored real time with the help of cloud computing, send alert or help messages whenever required. Accuracy of the data and availability of high speed network is the drawback in this model. In VithU app, which is an emergency app, it will send emergency help messages, with a link to the location where the user is situated, when the power button of the smartphone is pushed twice in a row. The text will be sent to the contacts stored in the app, every two minutes. But the button can be pressed unknowingly by children and can cause panic and also mobile needs to be in hand during emergency, which won't be the case in most of the cases. Pulicherla gowtami, Et. Al[2], developed a GSM based system, which will alert the contacts in case of theft or accident of vehicles. Chetal Indurwade, Et. Al [3], developed an app which helps to send danger messages to a group or individual. This has the drawback that mobile and network must be available at the emergency situations.

Rachel F Adler Et. al [4] have briefed about human multitasking in their paper. Humans do multiple jobs,time and again as a result of self fashioned distractions. These self-interruptions occur during nonappearance of external triggers such as email notifications or other electronic alerts. More self-interruptions result in poorer accuracy in all duties. Thus humans behave differently to different situations, so detecting the behavior during harassment will help to alert the concerned authorities. Deepak Akkil Et. al [5], proposes look alertness and active watch communications as substitute to interact with smart watches instead of touch screens or press buttons. It elaborates an experiment to demonstrate the user preferences for visual and handicapped feedback on a "glance" at the wrist watch. Results showed that user's choice differs depending on the complication of the communication. D. S. Quintana, Et. Al.[6] uses Heart rate variability (hrv) which indicate the variations of heart rate with respect to an average heart rate. For an average heart rate of 60 beats per minute (bpm), the interval between successive beats would not necessarily be exactly $1.0 \mathrm{sec}$, it may fluctuate from $0.5 \mathrm{sec}$ upto $2.0 \mathrm{sec}$. if the beat variations, changes at a given rate, this may be a symbol of a disparity in your training, load or stress.

\section{PROPOSED SYSTEM \& RESULTS}

The technology of detecting fingerprint data is achieved by digital imaging. This helps in reading, storing and analyzing fingerprint data. The fingerprint scanner embeds an optical or a capacitance reader which consists of the charge coupled device. This device comprises of light responsive diodes which give electric signals when illuminated. The small specs that hit the point the point are measured as pixels and from the group of pixels evolve the image. While placing the finger on the scanner's glass platter or observing surface, the camera captures a picture of

Revised Manuscript Received on August 14, 2019.

Pheba Cherian, Saveetha Engineering College, Chennai, Tamilnadu, India(email: phebacherian@gmail.com) India

A. Deepak, Saveetha School of Engineering, Chennai, Tamilnadu, 


\section{A SMART WATCH FOR WOMEN SECURITY}

the finger impressions by illumination. Figure 1, shows the structure of the fingerprint acquirement using ocular scanner and the image at the right side is the real time illustration of the machine.

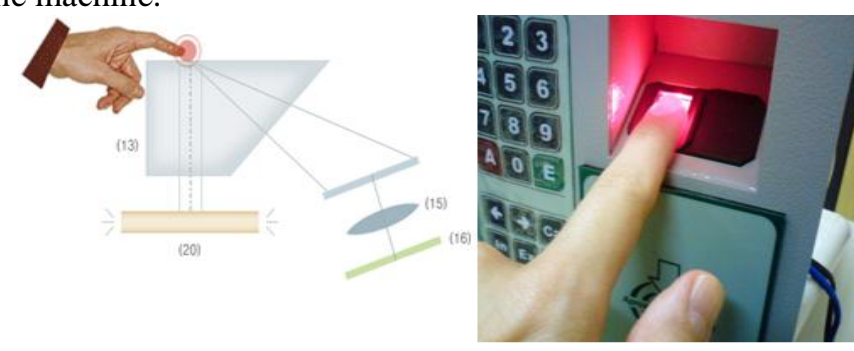

Figure 1: Fingerprint scanner

The detected image may contain relevant and irrelevant characteristics. By using thresholding, the image is filtered. The pixels whose gray level value or intensity, lesser than the threshold are eliminated whereas those with intensity greater than the threshold are chosen. Normalization is done to obtain a uniform pattern, so that the image pixels should have only desired boundaries of gray values. The gradient of each and every pixel is calculated at $\mathrm{x}$ and $\mathrm{y}$ directions to properly align the image and then the average of vector orthogonal to the gradient are determined to calculate the orientation. In order to construct frequency image, the gray values of each pixel is projected along the direction perpendicular to the orientation of the ridges and the number of pixels between consecutive minimums in the signal, which correspond to the rim is calculated. It can also be found using fourier transform technique. Unwanted noise is removed by filtering the image using Gabor or Butterworth filter. Thresholding techniques are used to convert the filtered image into binary image to improve the contrast. Image thinning is performed to eliminate forefront pixels to one pixel wide. It is then matched with previously stored images in the device.

To connect external network, ESP8266, WIFI module is used. Figure 2, shows a heart beat sensor, which gives digital output with respect to the hearts rhythm, when the finger is positioned. The LED blazes in accordance with the heartbeat. This output signal is measured and displayed as beats per minute using a microcontroller. The principle of working includes light modulation due to flow of blood during each pulse through the finger. It consists of a light sensing part and a red LED. The LED must be very bright so that the light should penetrate through the finger and detect the blood flow. Blood flows through the blood vessels, when it is pumped from the heart. The finger becomes more obscure and so less light will cross the finger and reach the detector. The detector collects different signal during each heart pulse and this is transformed to electrical pulse. This signal is activated and amplified and outputs as logic high level signal. An LED is also incorporated which flicker on each heart beat.

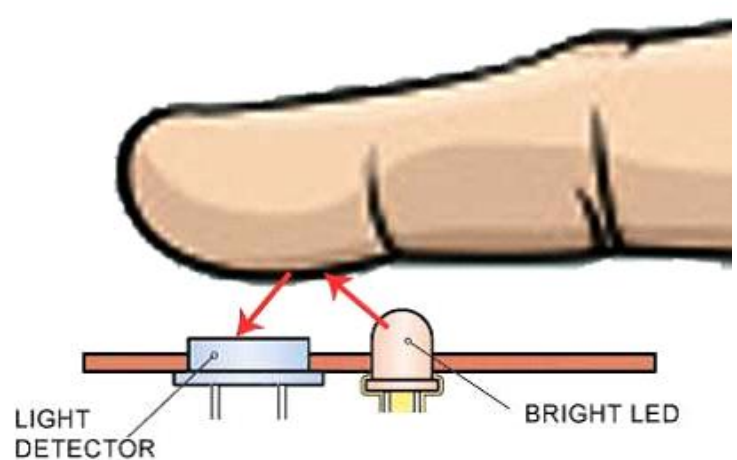

Figure 2: Heartbeat Sensor

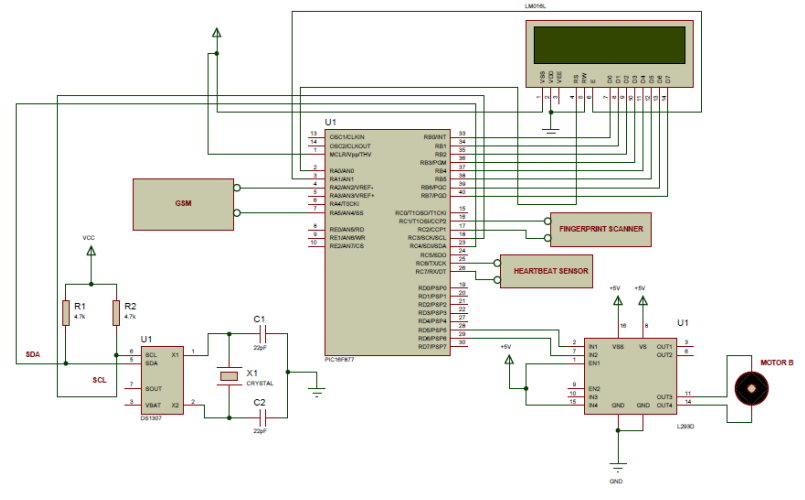

Figure 3: Implementation

\section{III: BLOCK DIAGRAM AND WORKING}

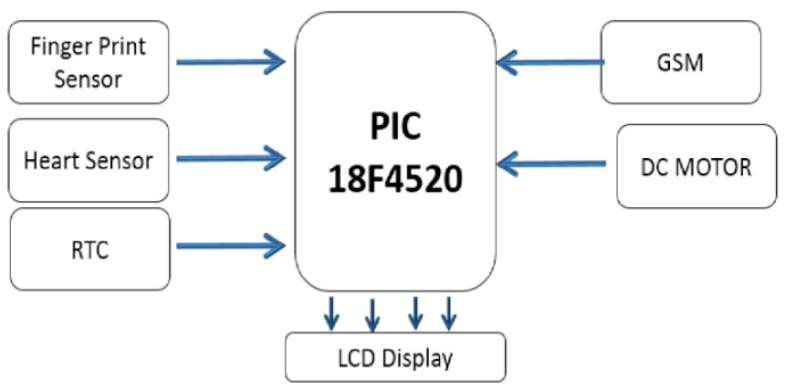

Figure 4: Block Diagram

Figure 4 , shows the block diagram of the device. The device is having the sensing unit for monitoring the heartbeat of the individual; an infrared (IR) sensor is used as heartbeat sensor. The IR sensor incorporates a light sensing part and a very intense red LED. When heart pumps, during each breath, the blood pass through the blood vessels, the finger becomes foggy to the detector and hence light which arrive at the detector will be less. With each heart pulse the different signals arrives at the detector. This signal deviation is converted to electrical pulse. This electrical signal is improved and activated through an amplifier which outputs as logic high level signal. The output signal is also indicated by another LED which flickers on each heartbeat. The normal heartbeat rate is pre-set, if any huge variation is found in the individual's heartbeat, the device sends the details to the user's mobile (via Wi-Fi or SMS or E-Mail). If any harassment takes place to women, naturally heartbeat will increase, then, the wearable device sends SMS to

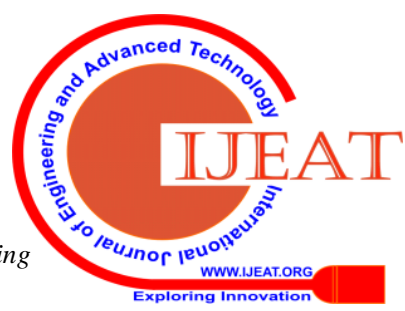


respective registered people or police through GSM technology. The system will take 10 seconds delay to send the SMS. Once the user places the finger in the fingerprint scanner the system understands that the person is normal and stops the SMS alert to respective people. Thus if the individual is in normal condition, it is acknowledged. The system consists of RTC (Real Time Clock) which will display the clock in the system. The fingerprint scanner is enabled for personalizing the device by providing high security so that only legitimate persons can operate the device. Also a custom-made lock is interfaced with fingerprint scanner which uses a DC motor for locking and unlocking. LCD (Liquid Crystal Display) screen is used as electronic display, which displays the date, time, registered user's mobile number and for few settings. RPS (Regulated Power Supply) is used as DC source of power to operate. Here in this proposed system, we use SMS alert enabled through GSM as medium of communication but it can be adopted using Internet access by wireless access point method. All the devices are interfaced to the PIC 18F4520.

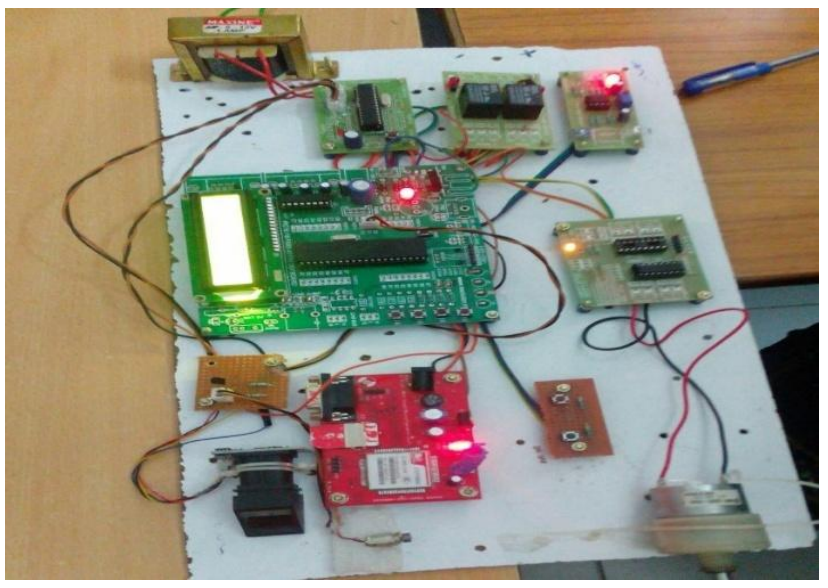

Figure 5: Hardware Implementation

\section{IV: CONCLUSION}

With the proposed system women security can be improved. A heartbeat sensor is attached to sense the beat rate of the person. If the heart beat increases a alert SMS is sent to the respective person. A fingerprint sensor is used to open and close the system by using the same finger.

A real time regulator is used to keep the current time updated every second. GSM technology is used to send the SMS. In case the problem is resolved, then the person can place the finger and the SMS will not be sent. In future the device can be improved by using micro fabricated sensors and actuators. Hence can be made available to the public at affordable prices and as a wearable device.

\section{REFERENCES}

1. International Conference on Recent Trends in Electronics, Information \& Communication Technology (RTEICT). IEEE, 2016.

2. Gowtami pulicherla, and Sridhar sunkari. "advanced system for vehicle and women safety."

3. Pande, Abhijit. "Helping Hands: An Android Based Women Security System." (2018).

4. Rachel F. et.al. Selfinterruptions in discretionary multitasking. Computers in Human Behavior, 29 (4). 1441-1449. DOI: 10.1016/j.chb.2013.01.040
5. Deepak Akkil et.al. Glance Awareness and Gaze Interaction in Smartwatches. in Proceedings of the 33rd Annual ACM Conference Extended Abstracts on Human Factors in Computing Systems, ACM, 1271-1276. DOI: 10.1145/2702613.2732816

6. Patrick Baudisch et.al 2009. Back-of-device interaction allows creating very small touch devices. in Proceedings of the SIGCHI Conference on Human Factors in Computing Systems, ACM, 1923-1932. DOI: 10.1145/1518701.1518995

7. Dennis B. Beringer. 1984. Caculator Watches as Data Entry Devices: The Fundamental Things Apply as Time Goes by. Proceedings of the Human Factors and Ergonomics Society Annual Meeting, 28 (3). 253-257. DOI: $10.1177 / 154193128402800313$

8. Jelmer P. Borst, Et.al,What Makes Interruptions Disruptive: A ProcessModel Account of the Effects of the Problem State Bottleneck on Task Interruption and Resumption. in Proceedings of the 33rd Annual ACM Conference on Human Factors in Computing Systems, ACM, Seoul, Republic of Korea, 2971-2980. DOI: 10.1145/2702123.2702156

9. Barry Brown, Moira McGregor and Eric Laurier. 2013 iPhone in vivo: video analysis of mobile device use. in Proceedings of the SIGCHI Conference on Human Factors in Computing Systems, ACM, DOI: $10.1145 / 2470654.2466132$ 